\title{
On classical and quantum deformations of gauge theories
}

\author{
I. L. Buchbinder ${ }^{1,2, a}$, P. M. Lavrov ${ }^{1,2, b}$ \\ ${ }^{1}$ Center of Theoretical Physics, Tomsk State Pedagogical University, Kievskaya St. 60, Tomsk 634061, Russia \\ ${ }^{2}$ National Research Tomsk State University, Lenin Av. 36, Tomsk 634050, Russia
}

Received: 30 August 2021 / Accepted: 17 September 2021 / Published online: 29 September 2021

(C) The Author(s) 2021

\begin{abstract}
We elaborate the generalizations of the approach to gauge-invariant deformations of the gauge theories developed in our previous work (Buchbinder and Lavrov in JHEP 06:097, 2021). In the given paper we construct the exact transformations defying the gauge-invariant deformed theory on the base of initial gauge theory with irreducible open gauge algebra. Like in [1], for the theories with open gauge algebras these transformations are the shifts of the initial gauge fields $A \rightarrow A+h(A)$, with the help of the arbitrary and in general non-local functions $h(A)$. The results are applied to study the quantum aspects of the deformed theories. We derive the exact relation between the quantum effective actions for the above classical theories, where one is obtained from another with the help of the deformation.
\end{abstract}

\section{Introduction}

The gauge theories play a key role in modern theoretical and mathematical physics. Therefore, the study of new classical and quantum aspects of gauge theories still deserves a close attention. One of such new aspects has been considered in our recent paper [1], where a gauge-invariant deformation of classical gauge theories was worked out. To be more precise, we have described a deformation of the classical gauge theory with a closed gauge algebra, which again leads to a gauge theory with a closed algebra. It was pointed out in [1] that this deformation can be local or non-local. In the first case the deformation results in a theory equivalent to the initial one, in the second case, we obtain a new gauge theory with new gauge algebra. In some case such a non-local theory posses a closed local sector, forming a new local gauge theory. As a result, the deformation under consideration can be treated as method to generate the new gauge theories based given gauge theories. In particular, it was shown in [1] that the

\footnotetext{
a e-mail: joseph@tspu.edu.ru

be-mail: lavrov@tspu.edu.ru (corresponding author)
}

Yang-Mills theory is obtained as the deformation of abelain gauge theory, and a cubic interaction vertex in bosonic higher spin theory is generated as the deformation of free bosonic higher spin theory.

Derivation of the deformation in [1] was based on the Batalin-Vilkovisky (BV) formalism [2-4], where the central object is the master equation, allowing to explore the classical and quantum aspects of gauge theories by some universal way. ${ }^{1}$ Construction of the gauge-invariant deformation is closely related with the study of the classical master equation. The solution to this equation was investigated in the works $[8,9]$ in the form of an expansion over some parameter and was shown that finding the solution reduces to cohomology problems. Applications of such an approach to Yang-Mills theory and higher spin field theory were considered in the works [10-14] respectively. Unlike all these works, our approach does not require to find a solution to the master equation in the form of series but allows to construct a general solution to master equation in closed form.

In this paper, we continue to explore the gauge-invariant deformation of the gauge theories and study the two new aspects. First, we generalize the results of the work [1] for gauge theories with irreducible generators and open gauge algebra. The examples of such theories are the supergravity models in component formulation, where the local superalgebras are only on-shell closed. ${ }^{2}$ Second, using the above results for classical deformations, we study the quantum aspects of the deformed theories and derive the exact relation between quantum effective actions of initial and deformed gauge theories.

The paper is organized as follows. In Sect. 2, we consider the basic notions of classical theory with irreducible

\footnotetext{
${ }^{1}$ See the recent developments and applications of the BV-formalism in [5-7] and the references therein.

${ }^{2}$ For some supergravity models there exists the superfield formulations where the local supersymmetry transformations form a closed algebra (see e.g. [15-17]). However for arbitrary supergravity models a formulation in terms of unconstrained superfields is still unknown.
} 
open gauge algebras within the BV-formalism. Section 3 is devoted to deriving the possible deformations of the initial classical gauge theory with the help of minimal anticanonical transformations. In Sect. 4 , we consider the quantization of the initial classical gauge theory and the gauge-invariant deformed theories using the BV-procedure and find the relationship between the effective action in these theories. In Sect. 5, we summarize the results.

In the paper we systematically use the DeWitt's condensed notations and employ the symbols $\varepsilon(A)$ for the Grassmann parity and $g h(A)$ for the ghost number, respectively. The right and left functional derivatives are marked by special symbols " $\leftarrow$ " and " $\rightarrow$ " respectively. Arguments of any functional are enclosed in square brackets [ ], and arguments of any function are enclosed in parentheses ( ).

\section{Classical gauge theory in the BV-formalism}

We consider a classical gauge theory of the fields $A=\left\{A^{i}\right\}$ with the Grassmann parities $\varepsilon\left(A^{i}\right)=\varepsilon_{i}$ and the ghost numbers $\operatorname{gh}\left(A^{i}\right)=0$. The theory is described by an initial action $S_{0}[A]$ invariant under the gauge transformations $\delta A^{i}=R_{\alpha}^{i}(A) \xi^{\alpha}$,

$S_{0, i}[A] R_{\alpha}^{i}(A)=0, \quad S_{0, i}[A]=S_{0}[A] \overleftarrow{\partial}_{A^{i}}$

where $R_{\alpha}^{i}(A)\left(\varepsilon\left(R_{\alpha}^{i}(A)\right)=\varepsilon_{i}+\varepsilon_{\alpha}, \operatorname{gh}\left(R_{\alpha}^{i}(A)\right)=0\right)$ are the gauge generators and the gauge parameters $\xi^{\alpha}\left(\varepsilon\left(\xi^{\alpha}\right)=\right.$ $\left.\varepsilon_{\alpha}\right)$ are the arbitrary functions of space-time coordinates. It is assumed that the gauge generators obey an irreducible and, generally speaking, open gauge algebra,

$$
\begin{gathered}
R_{\alpha, j}^{i}(A) R_{\beta}^{j}(A)-(-1)^{\varepsilon_{\alpha} \varepsilon_{\beta}} R_{\beta, j}^{i}(A) R_{\alpha}^{j}(A) \\
=-R_{\gamma}^{i}(A) F_{\alpha \beta}^{\gamma}(A)-S_{0, j} M_{\alpha \beta}^{j i}(A),
\end{gathered}
$$

where $R_{\alpha, j}^{i}(A)=R_{\alpha}^{i}(A) \overleftarrow{\partial}_{A^{j}}, F_{\alpha \beta}^{\gamma}(A)\left(\varepsilon\left(F_{\alpha \beta}^{\gamma}(A)\right)=\varepsilon_{\alpha}+\right.$ $\left.\varepsilon_{\beta}+\varepsilon_{\gamma}, \operatorname{gh}\left(F_{\alpha \beta}^{\gamma}(A)\right)=0\right)$ are the structure coefficients depending, in general, on the fields $A^{i}$, with the following symmetry properties $F_{\alpha \beta}^{\gamma}(A)=-(-1)^{\varepsilon_{\alpha} \varepsilon_{\beta}} F_{\beta \alpha}^{\gamma}(A)$. The functions $M_{\alpha \beta}^{i j}(A)$ satisfy the conditions $M_{\alpha \beta}^{i j}(A)=$ $-(-1)^{\varepsilon_{i} \varepsilon_{j}} M_{\alpha \beta}^{j i}(A)=-(-1)^{\varepsilon_{\alpha} \varepsilon_{\beta}} M_{\beta \alpha}^{i j}(A)$. If $M_{\alpha \beta}^{i j}(A) \neq 0$, then the gauge algebra is called open. Otherwise, the algebra is called closed. If $M_{\alpha \beta}^{i j}(A)=0$, and $F_{\alpha \beta}^{\gamma}$ does not depend on the fields, the gauge transformations form a gauge group and define a Lie algebra.

Following the BV-formalism [2,3], we introduce the minimal antisymplectic space of fields $\phi_{m}^{A}=\left(A^{i}, C^{\alpha}\right)$ and antifields $\phi_{m A}^{*}=\left(A_{i}^{*}, C_{\alpha}^{*}\right)$. Here $C^{\alpha}\left(\varepsilon\left(C^{\alpha}\right)=\varepsilon_{\alpha}+\right.$ $\left.1, \operatorname{gh}\left(C^{\alpha}\right)=1\right)$ are the ghost fields. The antifields $\phi_{m A}^{*}$ obey the following properties

$\varepsilon\left(\phi_{m A}^{*}\right)=\varepsilon\left(\phi_{m}^{A}\right)+1, \quad \operatorname{gh}\left(\phi_{m A}^{*}\right)=-1-\operatorname{gh}\left(\phi_{m}^{A}\right)$.
The basic object of the BV-formalism is the extended action $S_{m}=S_{m}\left[\phi_{m}, \phi_{m}^{*}\right]$ satisfying the classical master equation, ${ }^{3}$

$\left(S_{m}, S_{m}\right)=0$,

and the boundary condition,

$\left.S_{m}\left[\phi_{m}, \phi_{m}^{*}\right]\right|_{\phi_{m}^{*}=0}=S_{0}[A]$.

The action $S_{m}$ up to the terms linear in antifields is written in the form

$$
\begin{aligned}
S_{m}= & S_{0}[A]+A_{i}^{*} R_{\alpha}^{i}(A) C^{\alpha}-\frac{1}{2} C_{\gamma}^{*} F_{\alpha \beta}^{\gamma}(A) C^{\beta} C^{\alpha}(-1)^{\varepsilon_{\alpha}} \\
& +O\left(\phi^{* 2}\right) .
\end{aligned}
$$

It is proved that the solutions to the classical master equation in the minimal antisymplectic space encode all structure functions of the gauge algebra of a given classical gauge theory $[2,3]$.

The next step of the BV-formalism [2,3] involves introduction of the full antisymplectic space of fields $\phi^{A}$ and antifields $\phi_{A}^{*}$. For irreducible gauge theories the set of fields and antifields reads

$\phi^{A}=\left(A^{i}, C^{\alpha}, \bar{C}^{\alpha}, B^{\alpha}\right), \quad \phi_{A}^{*}=\left(A_{i}^{*}, C_{\alpha}^{*}, \bar{C}_{\alpha}^{*}, B_{\alpha}^{*}\right)$,

where $\bar{C}^{\alpha}\left(\varepsilon\left(\bar{C}^{\alpha}\right)=1, \operatorname{gh}\left(\bar{C}^{\alpha}\right)=-1\right)$ are the anti-ghost fields and $B^{\alpha}\left(\varepsilon\left(B^{\alpha}\right)=0, \operatorname{gh}\left(B^{\alpha}\right)=0\right)$ are the auxiliary (Nakanishi-Lautrup) fields. In the space of the fields (7) the action $S=S\left[\phi, \phi^{*}\right]$

$S=S_{m}+\bar{C}_{\alpha}^{*} B^{\alpha}$

is introduced. This action satisfies the classical master equation

$(S, S)=0 \quad$ with $\left.S\left[\phi, \phi^{*}\right]\right|_{\phi^{*}=0}=S_{0}[A]$.

The gauge invariance of the initial action $S_{0}[A]$ leads to invariance of the action $S=S\left[\phi, \phi^{*}\right]$,

$\delta_{B} S=0$

under the BRST transformations [18, 19]

$\delta_{B} \phi^{A}=\left(\phi^{A}, S\right) \mu=\vec{\partial}_{\phi_{A}^{*}} S \mu, \quad \delta_{B} \phi_{A}^{*}=0$.

As a consequence of these relations, the $S$ satisfies the classical master equation. Here $\mu$ is a constant Grassmann parameter.

It is proved that there exist the specific transformations of fields and antifields, preserving the antibracket, which are called anticanonical $[2,3]$. It means that making an arbitrary

\footnotetext{
${ }^{3}$ For any set of fields $\phi^{A}$ and antifields $\phi_{A}^{*}$ and arbitrary functionals $G=G\left[\phi, \phi^{*}\right]$ and $H=H\left[\phi, \phi^{*}\right]$ the antibracket is defined by the rule $(G, H)=G\left(\overleftarrow{\partial}_{\phi^{A}} \vec{\partial}_{\phi_{A}^{*}}-\overleftarrow{\partial}_{\phi_{A}^{*}} \vec{\partial}_{\phi^{A}}\right) H$
} 
anticanonical transformation in solution to the classical master equation, we obtain an action satisfying again to the classical master equation. Namely, this fact was used to construct a gauge-invariant deformation of the gauge theories [1].

\section{Gauge-invariant deformation of classical theory}

Gauge invariant deformation of the classical gauge theory was stated in our paper [1] for the theory with closed gauge algebra. Now we generalize the results of [1] for the theory where gauge algebra is open.

According to the results of the paper [1], the deformation of initial theory in the full antisymplectic space is described by the following anticanonical transformations of the solution (8),

$\phi_{A}^{*}=Y\left[\phi, \Phi^{*}\right] \overleftarrow{\partial}_{\phi^{A}}, \quad \Phi^{A}=\vec{\partial}_{\Phi_{A}^{*}} Y\left[\phi, \Phi^{*}\right]$

where $Y=Y\left[\phi, \Phi^{*}\right](\varepsilon(Y)=1, \operatorname{gh}(Y)=-1)$ is the generating functional of the anticanonical transformation. These transformations are non-trivial in the sector of minimal antisymplectic space only and, in general, are described by the generating functional of the form ${ }^{4}$

$Y\left[\phi, \Phi^{*}\right]=\Phi_{A}^{*} \phi^{A}+\mathcal{A}_{i}^{*} h^{i}(A)+\mathcal{C}_{\alpha}^{*} g_{\beta}^{\alpha}(A) C^{\beta}$,

where $h^{i}(A)\left(\varepsilon\left(h^{i}(A)\right)=\varepsilon_{i}, \operatorname{gh}\left(h^{i}(A)\right)=0\right)$ and $g_{\beta}^{\alpha}(A)$ $\left(\varepsilon\left(g_{\beta}^{\alpha}(A)\right)=\varepsilon_{\alpha}+\varepsilon_{\beta}, \operatorname{gh}\left(g_{\beta}^{\alpha}(A)\right)=0\right)$ are the arbitrary functions of fields $A^{i}$. Here the following notations

$\Phi^{A}=\left(\mathcal{A}^{i}, \mathcal{C}^{\alpha}, \overline{\mathcal{C}}^{\alpha}, \mathcal{B}^{\alpha}\right), \quad \Phi_{A}^{*}=\left(\mathcal{A}_{i}^{*}, \mathcal{C}_{\alpha}^{*}, \overline{\mathcal{C}}_{\alpha}^{*}, \mathcal{B}_{\alpha}^{*}\right)$

are used.

It was proved in [1] that the anticanonical transformations (13) of the initial classical action $S_{0}[A]$ lead to the deformed action $\widetilde{S}_{0}[A]$ of the form

$\widetilde{S}_{0}[A]=S_{0}[A+h(A)]$.

It is worth emphasizing that the deformed action (15) has extremely simple form and is described by the functions $h^{i}(A)$ only. The action $\widetilde{S}_{0}[A]$ is invariant under gauge transformations, $\delta A^{i}=\widetilde{R}_{\alpha}^{i}(A) \xi^{\alpha}$,

$\widetilde{S}_{0, i}[A] \widetilde{R}_{\alpha}^{i}(A)=0$.

Here

$\widetilde{R}_{\alpha}^{i}(A)=\left(M^{-1}(A)\right)_{j}^{i} R_{\alpha}^{j}(A+h(A))$

are deformed gauge generators and the $\left(M^{-1}(A)\right)^{i}{ }_{j}$ is inverse to the matrix

$M_{j}^{i}(A)=\delta^{i}{ }_{j}+h^{i}(A) \overleftarrow{\partial}_{A^{j}}$

\footnotetext{
${ }^{4}$ Note that the form of transformations (13) is the same both for closed and for open gauge algebras.
}

The deformed gauge generators (17) are formulated in terms of functions $h^{i}(A)$ only. The functions $g_{\beta}^{\alpha}(A)$ are responsible for possible transformations of ghost fields $C^{\alpha}$ and antifields $C_{\alpha}^{*}$, which do not touch the deformations in the sector of fields $A^{i}$. It allows us to introduce the minimal anticanonical transformations describing by the generating functional

$Y_{\min }\left[\phi, \Phi^{*}\right]=\Phi_{A}^{*} \phi^{A}+\mathcal{A}_{i}^{*} h^{i}(A)$.

In this case the procedure of gauge-invariant deformations of initial gauge-invariant theory is controlled by the functions $h^{i}(A)$ only. It means that further there is no need to use the functions $g_{\beta}^{\alpha}(A)$. As a result, the gauge-invariant deformations corresponds to the following non-trivial change of variables

$$
A^{i} \rightarrow A^{i}+h^{i}(A), \quad A_{i}^{*} \rightarrow A_{j}^{*}\left(M^{-1}(A)\right)_{i}^{j} .
$$

The gauge algebra of deformed generators (17) can be calculated the same way as it was done in the paper [1], but taking into account that the initial gauge algebra is now open. The result has the form

$$
\begin{aligned}
& \widetilde{R}_{\alpha, j}^{i}(A) \widetilde{R}_{\beta}^{j}(A)-(-1)^{\varepsilon_{\alpha} \varepsilon_{\beta}} \widetilde{R}_{\beta, j}^{i}(A) \widetilde{R}_{\alpha}^{j}(A) \\
& \quad=-\widetilde{R}_{\gamma}^{i}(A) \widetilde{F}_{\alpha \beta}^{\gamma}(A)-\widetilde{S}_{0, j}[A] \widetilde{M}_{\alpha \beta}^{j i}(A),
\end{aligned}
$$

Here $\widetilde{F}_{\alpha \beta}^{\gamma}(A)=F_{\alpha \beta}^{\gamma}(A+h(A))$ are the deformed structure functions and the functions $\tilde{M}_{\alpha \beta}^{j i}(A)$ are

$$
\begin{aligned}
\tilde{M}_{\alpha \beta}^{j i}(A)= & \left(M^{-1}(A)\right)_{l}^{j}\left(M^{-1}(A)\right)_{k}^{i} M_{\alpha \beta}^{k l}(A+h(A)) \\
& \times(-1)^{\varepsilon_{l}\left(\varepsilon_{i}+\varepsilon_{k}\right)} .
\end{aligned}
$$

The symmetry properties of deformed structures $\widetilde{F}_{\alpha \beta}^{\gamma}(A)$ and $\widetilde{M}_{\alpha \beta}^{j i}(A)$ coincide with corresponding ones of nondeformed structures. The same is valid for the Grassmann parities and ghost numbers. We see that the deformed algebra is again irreducible and open.

After the anticanonical transformations (19), the action (8) transforms into the functional $\widetilde{S}=\widetilde{S}\left[\phi, \phi^{*}\right]$ which satisfies the classical master equation

$(\widetilde{S}, \widetilde{S})=0$

and has the following form up to the terms linear in antifields

$$
\begin{aligned}
\widetilde{S}= & \widetilde{S}_{0}[A]+A_{i}^{*} \widetilde{R}_{\alpha}^{i}(A) C^{\alpha}-\frac{1}{2} C_{\gamma}^{*} \widetilde{F}_{\alpha \beta}^{\gamma}(A) C^{\beta} C^{\alpha}(-1)^{\varepsilon_{\alpha}} \\
& +\bar{C}_{\alpha}^{*} B^{\alpha}+O\left(\phi^{* 2}\right)
\end{aligned}
$$

The action (24) is invariant under the BRST transformations,

$$
\delta_{B} \widetilde{S}=0, \quad \delta_{B} \phi^{A}=\left(\phi^{A}, \widetilde{S}\right) \mu=\vec{\partial}_{\phi_{A}^{*}} \widetilde{S} \mu, \quad \delta_{B} \phi_{A}^{*}=0,
$$


due to the Eq. (23). As a result, we have described the gaugeinvariant deformation of classical gauge theory for the case of open gauge algebra. If the initial gauge algebra is closed, the result (21) is reduced to deformed gauge algebra constructed in the paper [1] for the special case $g_{\beta}^{\alpha}(A)=0$.

\section{Deformation of quantum gauge theory}

This section derives the general relationship between the quantum effective actions of two classical gauge theories obtained from each other using the deformation procedure described in the previous section. To be more precise, we will consider the effective actions of two such theories in the sector of fields $A^{i}$ only.

According to BV-formalism [2,3], the generating functional of Green functions in the case under consideration is given by the following functional integral

$$
Z[j]=\int D \phi \exp \left\{\frac{i}{\hbar}\left(S_{g}[\phi]+j A\right)\right\},
$$

where $D \phi=D A D C D \bar{C} D B$ and

$S_{g}[\phi]=\left.S\left[\phi, \phi^{*}\right]\right|_{\phi^{*}=\psi[\phi] \overleftarrow{\partial_{\phi}}}$

is the gauge fixed action. Here $j=\left\{j_{i}\right\}\left(\varepsilon\left(j_{i}\right)=\right.$ $\left.\varepsilon_{i}, \operatorname{gh}\left(j_{i}\right)=0\right)$ is the set of external sources to the fields $A^{i}, \psi[\phi](\varepsilon(\psi[\phi])=1, \operatorname{gh}(\psi[\phi])=1)$ is the gauge fixing functional which is chosen standardly as $\psi[\phi]=$ $\bar{C}^{\alpha} \chi_{\alpha}(A)$, where $\chi_{\alpha}(A)\left(\varepsilon\left(\chi_{\alpha}(A)\right)=\varepsilon_{\alpha}, \operatorname{gh}\left(\chi_{\alpha}(A)\right)=0\right)$ are the gauge fixing functions lifting the degeneracy of action $S\left[\phi, \phi^{*}\right]$. It allows to write

$$
\begin{aligned}
S_{g}[\phi]= & S_{0}[A]+\bar{C}^{\alpha} \chi_{\alpha, i}(A) R_{\beta}^{i}(A) C^{\beta} \\
& +\chi_{\alpha}(A) B^{\alpha}+O\left(C^{2}\right) .
\end{aligned}
$$

For Yang-Mills type gauge theories the first three terms in the r.h.s of (28) present the well known Faddeev-Popov action [20].

According to the same procedure, the generating functional of Green functions of the deformed theory has the form

$\widetilde{Z}[j]=\int D \phi \exp \left\{\frac{i}{\hbar}\left(\widetilde{S}_{g}[\phi]+j A\right)\right\}$,

where

$\widetilde{S}_{g}[\phi]=\left.\widetilde{S}\left[\phi, \phi^{*}\right]\right|_{\phi^{*}=\widetilde{\psi}[\phi] \overleftarrow{\partial_{\phi}}}$

is the gauge fixed action for deformed theory corresponding to the gauge fixing condition subjected to the change of fields $A^{i}$ coming from the minimal anticanonical transformations

$\widetilde{\psi}[\phi]=\psi[A+h(A), \bar{C}]$.
In this case, we have the simple relation

$\widetilde{S}_{g}[\phi]=\left.S_{g}[\phi]\right|_{A \rightarrow A+h(A)}$

and, therefore,

$$
\begin{aligned}
\widetilde{S}_{g}[\phi]= & \widetilde{S}_{0}[A]+\bar{C}^{\alpha} \widetilde{\chi}_{\alpha, i}(A) \widetilde{R}_{\beta}^{i}(A) C^{\beta} \\
& +\tilde{\chi}_{\alpha}(A) B^{\alpha}+O\left(C^{2}\right) .
\end{aligned}
$$

Let us make in the functional integral (26) the change of variables

$A^{i} \rightarrow A^{i}+h^{i}(A)$,

and take into account the relation (32) as well as the Jacobian $J[A]$ corresponding to the transformation (34)

$J[A]=\operatorname{sDet} M(A)=\exp \{\mathrm{s} \operatorname{Tr} \ln M(A)\}$,

where the symbols sDet and sTr mean the functional superdeterminant and functional supertrace respectively. After that we arrive at the relation

$Z[j]=: \exp \left\{\frac{i}{\hbar} j h(\widehat{q})\right\}: \exp \{\operatorname{sTr} \ln M(\widehat{q})\} \widetilde{Z}[j]$.

The relation (36) allows to express the Green functions of the initial theory through the Green functions in the deformed theory. In the equation (36) the matrix $M(A)$ is defined in (18) and the notation

$\widehat{q}^{i}=\frac{\hbar}{i} \frac{\vec{\delta}}{\delta j_{i}}$

is used. The symbol : : means

$: \exp \{j \widehat{q}\}:=\sum_{n=0}^{\infty} \frac{1}{n !} j_{1} j_{2} \cdots j_{n} \widehat{q}_{n} \cdots \widehat{q}_{2} \widehat{q}_{1}$,

i.e all operators $\widehat{q}^{i}$ must stand from the right of sources $j_{i}$ in the order indicated exactly in (38).

Also we can find the relation inverse to (36). To do this let us act by the operator : $\exp \{(i / \hbar) j h(\widehat{q})\}:$ on (29) from the left side. It leads to

$$
\begin{aligned}
& : \exp \left\{\frac{i}{\hbar} j h(\widehat{q})\right\}: \widetilde{Z}[j] \\
& \quad=\int D \phi \exp \left\{\frac{i}{\hbar}\left(\widetilde{S}_{g}[\phi]+j A+j h(A)\right)\right\} .
\end{aligned}
$$

Making use in the functional integral (39) the change of variables

$A^{i}+h^{i}(A) \rightarrow A^{i}$

we obtain

$: \exp \left\{\frac{i}{\hbar} j h(\widehat{q})\right\}: \widetilde{Z}[j]=\exp \{-\operatorname{sTr} \ln M(\widehat{q})\} Z[j]$. 
Finally, the relation inverse to (36) reads

$\widetilde{Z}[j]=: \exp \left\{-\frac{i}{\hbar} j h(\widehat{q})\right\}: \exp \{-\operatorname{sTr} \ln M(\widehat{q})\} Z[j]$.

Next step of consideration is to introduce the generating functionals of the connected Green functions

$W[j]=-i \hbar \ln Z[j], \quad \widetilde{W}[j]=-i \hbar \ln \widetilde{Z}[j]$.

From the Eq. (36) it follows

$$
\begin{aligned}
W[j]= & \widetilde{W}[j]-i \hbar \ln \left(: \exp \left\{\frac{i}{\hbar} j h(\widehat{\widetilde{Q}})\right\}\right. \\
& : \exp \{\sin \ln M(\widehat{\widetilde{Q}})\}) \cdot 1 .
\end{aligned}
$$

where the operators $\widehat{\widetilde{Q}^{i}}$ are

$\widehat{\widetilde{Q}^{i}}=\exp \left\{-\frac{i}{\hbar} \widetilde{W}[j]\right\} \widehat{q}^{i} \exp \left\{\frac{i}{\hbar} \widetilde{W}[j]\right\}=\widehat{q}^{i}+\frac{\vec{\delta} \widetilde{W}[j]}{\delta j_{i}}$

Here the following commutation relations

$\left[q^{i}, q^{j}\right]=0 \quad \rightarrow \quad\left[\widehat{Q}^{i}, \widehat{Q}^{j}\right]=0$

were used in deriving the Eq. (44). In its turn the Eq. (42) in terms of functionals $W[j]$ and $\widetilde{W}[j]$ rewrites in the form

$$
\begin{aligned}
\widetilde{W}[j]= & W[j]-i \hbar \ln \left(: \exp \left\{-\frac{i}{\hbar} j h(\widehat{Q})\right\}\right. \\
& : \exp \{-\operatorname{sTr} \ln M(\widehat{Q})\}) \cdot 1,
\end{aligned}
$$

where

$\widehat{Q}^{i}=\exp \left\{-\frac{i}{\hbar} W[j]\right\} \widehat{q}^{i} \exp \left\{\frac{i}{\hbar} W[j]\right\}=\widehat{q}^{i}+\frac{\vec{\delta} W[j]}{\delta j_{i}}$.

One can check that the relation $\left[\widehat{Q}^{i}, \widehat{Q}^{j}\right]=0$ is fulfilled as it should be.

Now one introduces the effective actions using the Legendre transformations of functionals $W[j]$ and $\widetilde{W}[j]$,

$$
\begin{gathered}
\Gamma[\mathcal{A}]=W[j]-j \mathcal{A}, \quad \mathcal{A}^{i}=\frac{\vec{\delta} W[j]}{\delta j_{i}}, \\
\Gamma[\mathcal{A}] \overleftarrow{\partial}_{\mathcal{A}^{i}}=-j_{i}, \\
\widetilde{\Gamma}[\widetilde{\mathcal{A}}]=\widetilde{W}[j]-j \widetilde{\mathcal{A}}, \quad \widetilde{\mathcal{A}}^{i}=\frac{\vec{\delta} \widetilde{W}[j]}{\delta j_{i}},
\end{gathered}
$$

$\widetilde{\Gamma}[\widetilde{\mathcal{A}}] \overleftarrow{\partial} \widetilde{\mathcal{A}}^{i}=-j_{i}$
Then, the relation (44) leads to the expressions

$$
\begin{aligned}
& \Gamma[\mathcal{A}]-\Gamma[\mathcal{A}] \overleftarrow{\partial}_{\mathcal{A}^{i}} \mathcal{A}^{i}=\widetilde{\Gamma}[\widetilde{\mathcal{A}}]-\widetilde{\Gamma}[\widetilde{\mathcal{A}}] \overleftarrow{\partial} \widetilde{\mathcal{A}}^{i} \widetilde{\mathcal{A}}^{i} \\
& -i \hbar \ln \left(: \exp \left\{-\frac{i}{\hbar}(\widetilde{\Gamma}[\widetilde{\mathcal{A}}] \overleftarrow{\partial} \widetilde{\mathcal{A}}) h(\widehat{\widetilde{\mathcal{A}}})\right\}\right. \\
& \quad: \exp \{\sin \ln M(\widehat{\widetilde{\mathcal{A}}})\}) \cdot 1
\end{aligned}
$$

where the notation

$\widetilde{\mathcal{A}^{i}}=\widetilde{\mathcal{A}}^{i}+i \hbar\left(\widetilde{\Gamma}^{(-1)}[\widetilde{\mathcal{A}}]\right)^{i j} \frac{\vec{\delta}}{\delta \widetilde{A}^{j}}$

is used. The matrix $\left(\widetilde{\Gamma}^{(-1)}[\widetilde{\mathcal{A}}]\right)^{i j}$ is inverse to the matrix

$$
\begin{aligned}
& (\widetilde{\Gamma}[\widetilde{\mathcal{A}}])_{i j}=\frac{\vec{\delta}}{\delta \mathcal{A}^{i}} \widetilde{\Gamma}[\widetilde{A}] \frac{\overleftarrow{\delta}}{\delta \mathcal{A}^{j}} \\
& (\widetilde{\Gamma}[\widetilde{\mathcal{A}}])_{i k}\left(\widetilde{\Gamma}^{(-1)}[\widetilde{\mathcal{A}}]\right)^{k j}=\delta_{i}{ }^{j}
\end{aligned}
$$

with the following symmetry properties

$$
\begin{array}{rlr}
\left(\widetilde{\Gamma}^{(-1)}[\tilde{\mathcal{A}}]\right)^{i j} & =-\left(\widetilde{\Gamma}^{(-1)}[\tilde{\mathcal{A}}]\right)^{j i}(-1)^{\left(\varepsilon_{i}+1\right)\left(\varepsilon_{j}+1\right)}, & (\widetilde{\Gamma}[\widetilde{\mathcal{A}}])_{i j} \\
& =-(\widetilde{\Gamma}[\widetilde{\mathcal{A}}])_{j i}(-1)^{\left(\varepsilon_{i}+1\right)\left(\varepsilon_{j}+1\right)} .
\end{array}
$$

Note that the operators $\widehat{\widetilde{\mathcal{A}^{i}}}$ commute,

$\left[\widetilde{\mathcal{A}}^{i}, \widetilde{\mathcal{A}}^{j}\right]=0$

due to the relations (54). Differentiating the relation (44) with respect to sources $j_{i}$ and taking into account the rule for derivative of the logarithm of operator $B(t)$ with respect to a parameter $t$,

$\partial_{t} \ln B(t)=B^{-1}(t) \partial_{t} B(t)$,

we find the equation

$$
\begin{aligned}
A^{i}= & \widetilde{A}^{i}+\left(: \exp \left\{-\frac{i}{\hbar}(\widetilde{\Gamma}[\widetilde{\mathcal{A}}] \overleftarrow{\partial} \widetilde{\mathcal{A}}) h(\widehat{\widetilde{\mathcal{A}}})\right\}\right. \\
& : \exp \{\operatorname{sTr} \ln M(\widehat{\widetilde{\mathcal{A}}})\})^{-1} \\
& \times\left(: \exp \left\{-\frac{i}{\hbar}(\widetilde{\Gamma}[\widetilde{\mathcal{A}}] \overleftarrow{\partial} \tilde{\mathcal{A}}) h(\widehat{\widetilde{\mathcal{A}}})\right\}\right. \\
& \left.: h^{i}(\widehat{\widetilde{\mathcal{A}}}) \exp \{\sin \ln M(\widehat{\widetilde{\mathcal{A}}})\}\right) \cdot 1,
\end{aligned}
$$


allowing to express the fields $A^{i}$ in terms of $\widetilde{A}^{i}$. Using this result in relation (51) we obtain the final expression

$$
\begin{aligned}
\Gamma[\mathcal{A}]= & \widetilde{\Gamma}[\widetilde{\mathcal{A}}]+\widetilde{\Gamma}[\widetilde{\mathcal{A}}] \overleftarrow{\partial} \widetilde{\mathcal{A}}^{i} \\
& \times\left(: \exp \left\{-\frac{i}{\hbar}(\widetilde{\Gamma}[\widetilde{\mathcal{A}}] \overleftarrow{\partial} \widetilde{\mathcal{A}}) h(\widehat{\widetilde{\mathcal{A}}})\right\}\right. \\
& : \exp \{\sin \ln M(\widehat{\widetilde{\mathcal{A}}})\})^{-1} \\
& \times\left(: \exp \left\{-\frac{i}{\hbar}(\widetilde{\Gamma}[\widetilde{\mathcal{A}}] \overleftarrow{\partial} \widetilde{\mathcal{A}}) h(\widehat{\widetilde{\mathcal{A}}})\right\}\right. \\
& \left.: h^{i}(\widehat{\widetilde{\mathcal{A}}}) \exp \{\operatorname{sTr} \ln M(\widehat{\widetilde{\mathcal{A}}})\}\right) \cdot 1 \\
& -i \hbar \ln \left(: \exp \left\{-\frac{i}{\hbar}(\widetilde{\Gamma}[\widetilde{\mathcal{A}}] \overleftarrow{\partial} \widetilde{\mathcal{A}}) h(\widehat{\widetilde{\mathcal{A}}})\right\}\right. \\
& : \exp \{\sin \ln M(\widetilde{\widetilde{\mathcal{A}}})\}) \cdot 1
\end{aligned}
$$

The expression (58) is a general relationship between the effective actions of two gauge theories obtained one from another by deformation procedure. Although the relation (58) looks complicated enough, it is the exact result in quantum gauge field theory. Turn attention to that that this relation is valid for the theories both with closed and open gauge algebras.

In many applications, it is sufficient to find the effective actions for the fields satisfying the equations of motion. Taking into account the possibility of such applications, one considers the obtained expression (58) on the equations of motion. The exact equations of motion for deformed effective action is written as follows

$\widetilde{\Gamma}[\widetilde{\mathcal{A}}] \overleftarrow{\partial}_{\widetilde{\mathcal{A}}^{i}}=0$

In this case the relation (58) reduces to the following simple enough form

$\Gamma[\mathcal{A}]=\widetilde{\Gamma}[\widetilde{A}]-i \hbar \operatorname{sTr} \ln M(\widehat{\widetilde{\mathcal{A}}}) \cdot 1$

Now one finds the expression for deformed effective action through effective action of initial theory for the fields on shell. First, find that inverse relations to (57)

$$
\begin{aligned}
\widetilde{A}^{i}= & A^{i}+\left(: \exp \left\{-\frac{i}{\hbar}\left(\Gamma[\mathcal{A}] \overleftarrow{\partial}_{\mathcal{A}}\right) h(\widehat{\mathcal{A}})\right\}\right. \\
& : \exp \{-\operatorname{sTr} \ln M(\widehat{\mathcal{A}})\})^{-1} \\
& \times\left(: \exp \left\{-\frac{i}{\hbar}\left(\Gamma[\mathcal{A}] \overleftarrow{\partial}_{\mathcal{A}}\right) h(\widehat{\mathcal{A}})\right\}\right. \\
& \left.: h^{i}(\widehat{\mathcal{A}}) \exp \{-\operatorname{sTr} \ln M(\widehat{\mathcal{A}})\}\right) \cdot 1
\end{aligned}
$$

Therefore

$$
\begin{aligned}
\widetilde{\Gamma}[\widetilde{\mathcal{A}}]= & \Gamma[\mathcal{A}]+\Gamma[\mathcal{A}] \overleftarrow{\partial}_{\mathcal{A}^{i}} \\
& \times\left(: \exp \left\{-\frac{i}{\hbar}\left(\Gamma[\mathcal{A}] \overleftarrow{\partial}_{\mathcal{A}}\right) h(\widehat{\mathcal{A}})\right\}: \exp \{\right. \\
& -\operatorname{sTr} \ln M(\widehat{\mathcal{A}})\})^{-1} \\
& \times\left(: \exp \left\{-\frac{i}{\hbar}\left(\Gamma[\mathcal{A}] \overleftarrow{\partial}_{\mathcal{A}}\right) h(\widehat{\mathcal{A}})\right\}: h^{i}(\widehat{\mathcal{A}}) \exp \{\right. \\
& -\operatorname{sTr} \ln M(\widehat{\mathcal{A}})\}) \cdot 1 \\
& -i \hbar \ln \left(: \exp \left\{-\frac{i}{\hbar}\left(\Gamma[\mathcal{A}] \overleftarrow{\partial}_{\mathcal{A}}\right) h(\widehat{\mathcal{A}})\right\}: \exp \{\right. \\
& -\operatorname{sTr} \ln M(\widehat{\mathcal{A}})\}) \cdot 1
\end{aligned}
$$

Here the notation

$\widehat{\mathcal{A}^{i}}=\mathcal{A}^{i}+i \hbar\left(\Gamma^{(-1)}[\mathcal{A}]\right)^{i j} \frac{\vec{\delta}}{\delta \mathcal{A}^{j}}$

is used and the operators $\widehat{\mathcal{A}^{i}}$ commute, $\left[\widehat{\mathcal{A}^{i}}, \widehat{\mathcal{A}^{j}}\right]=0$. The matrix $\left(\Gamma^{(-1)}[\mathcal{A}]\right)^{i j}$ is inverse to the matrix

$\Gamma[\mathcal{A}]_{i j}=\frac{\vec{\delta}}{\delta \mathcal{A}^{i}} \Gamma[\mathcal{A}] \frac{\overleftarrow{\delta}}{\delta \mathcal{A}^{j}}, \quad \Gamma[\mathcal{A}]_{i k}\left(\Gamma^{(-1)}[\mathcal{A}]\right)^{k j}=\delta_{i}{ }^{j}$

with the following symmetry properties

$$
\begin{array}{rlr}
\left(\Gamma^{(-1)}[\mathcal{A}]\right)^{i j} & =-\left(\Gamma^{(-1)}[\mathcal{A}]\right)^{j i}(-1)^{\left(\varepsilon_{i}+1\right)\left(\varepsilon_{j}+1\right)}, & \Gamma[\mathcal{A}]_{i j} \\
& =-\Gamma[\mathcal{A}]_{j i}(-1)^{\left(\varepsilon_{i}+1\right)\left(\varepsilon_{j}+1\right)} .
\end{array}
$$

On the extremals of the initial effective action,

$\Gamma[\mathcal{A}] \frac{\overleftarrow{\delta}}{\delta \mathcal{A}^{i}}=0$

the Eq. (63) takes the final simple enough form

$\widetilde{\Gamma}[\widetilde{\mathcal{A}}]=\Gamma[\mathcal{A}]+i \hbar \sin \ln M(\widehat{\mathcal{A}})\} \cdot 1$

Let us briefly discuss the relation of the result (69) with $S$-matrix structure of the deformed theory. As is known, the construction of the S-matrix in quantum field theory uses the effective action calculated on its extremals. If gaugeinvariant deformation of an initial action is performed with the help of a local function $h(A)$ then the matrix $M(A)$ is local as well. The second term in the r.h.s. (69) up to the numerical constant $i \hbar$ is the exponent of superdeterminant of a local function. Such superdeterminant is proportional to $\delta(0)$ which can be dropped off in the dimensional regularization because of $\delta(0)=0$. In this case the effective actions coincide, $\widetilde{\Gamma}[\widetilde{\mathcal{A}}]=\Gamma[\mathcal{A}]$. The same is valid for S-matrices. In general, when the function $h(A)$ involves the derivatives and/or it is non-local, the initial and deformed theories are not equivalent both at classical and quantum levels. 
As a result, we derived the exact relation between quantum effective actions of the two classical gauge theories obtained one from another by the deformation preserving the gauge invariance.

\section{Conclusion}

Let us summarize the results. We have generalized the approach to constructing the gauge-invariant deformations of a given classical gauge theory proposed in our previous work [1]. Unlike this work, we described the gauge-invariant deformation for the classical theories with open gauge algebra. Deformation of the initial action both for the theories with closed gauge algebras and for the theories with open gauge algebras is generated by the transformation of the fields $A^{i} \rightarrow A^{i}+h^{i}(A)$ with the arbitrary functions $h^{i}(A)$. If these functions are local and do not contain the derivatives, the deformed theory is classically equivalent to the initial theory. However, such functions can contain the derivatives or to be non-local. Then the deformation yields a higher derivative or non-local gauge theory. In the work [1], we argued that the non-local theories, obtained this way, can have a closed local sector. As a result, the deformation under construction can be treated as a procedure to create the new gauge theories on the base of the given gauge theories. Deformations of action and gauge transformations are generated by arbitrary functions $h^{i}(A)$, which are completely arbitrary. Explicit forms of the deformed generators and the deformed gauge algebra are given by the relations (17) and (21). If the initial gauge algebra is closed, the relation (21) is reduced to expression for deformed algebra in work [1].

Considering the two arbitrary gauge theories, where one is obtained from another by deformation procedure, we have derived a relation between quantum effective actions for such theories. This relation is given by the expression (58). Although the obtained relation looks complicated enough, it is an exact relation. Also, we proved that the relation (58) is essentially simplified on the extremals of the effective action. In our previous work [1], we constructed some examples of how the non-trivial gauge theories can be constructed by deformation of the free gauge theories. Taking into account these results, one can hope that the relation (58) can be used to relate the quantum aspects of the trivial and non-trivial gauge theories.

We emphasize that all the results above are based only on the general properties of gauge theories, and they do not appeal to specific details of concrete theories. In essence, they represent the exact relations in the classical and quantum theory of gauge fields.

Acknowledgements The work is supported by the Ministry of Education of the Russian Federation, project FEWF-2020-0003.
Data Availability Statement This manuscript has no associated data or the data will not be deposited. [Authors' comment: This is a purely theoretical study and no experimental data has been used, and do not need to be quoted.]

Open Access This article is licensed under a Creative Commons Attribution 4.0 International License, which permits use, sharing, adaptation, distribution and reproduction in any medium or format, as long as you give appropriate credit to the original author(s) and the source, provide a link to the Creative Commons licence, and indicate if changes were made. The images or other third party material in this article are included in the article's Creative Commons licence, unless indicated otherwise in a credit line to the material. If material is not included in the article's Creative Commons licence and your intended use is not permitted by statutory regulation or exceeds the permitted use, you will need to obtain permission directly from the copyright holder. To view a copy of this licence, visit http://creativecomm ons.org/licenses/by/4.0/.

Funded by SCOAP 3 .

\section{References}

1. I.L. Buchbinder, P.M. Lavrov, On a gauge-invariant deformation of a classical gauge-invariant theory. JHEP 06, 097 (2021). arXiv:2104.11930 [hep-th]

2. I.A. Batalin, G.A. Vilkovisky, Gauge algebra and quantization. Phys. Lett. B 102, 27-31 (1981)

3. I.A. Batalin, G.A. Vilkovisky, Quantization of gauge theories with linearly dependent generators. Phys. Rev. D 28, 2567-2582 (1983)

4. I.A. Batalin, G.A. Vilkovisky, Closure of the gauge algebra, generalized Lie algebra equations and Feynman rules. Nucl. Phys. B 234, 106 (1984)

5. I.A. Batalin, P.M. Lavrov, I.V. Tyutin, Finite anticanonical transformations in field-antifield formalism. Eur. Phys. J. C 75, 270 (2015). arXiv:1501.07334 [hep-th]

6. I.A. Batalin, P.M. Lavrov, Closed description of arbitrariness in resolving quantum master equation. Phys. Lett. B 758, 54-58 (2016). arXiv:1604.01888 [hep-th]

7. I.A. Batalin, P.M. Lavrov, I.V. Tyutin, Anticanonical transformations and Grand Jacobian. arXiv:2011.06429 [hep-th]

8. G. Barnich, M. Henneaux, Consistent coupling between fields with gauge freedom and deformation of master equation. Phys. Lett. B 311, 123-129 (1993). arXiv:hep-th/9304057

9. M. Henneaux, Consistent interactions between gauge fields: The cohomological approach. Contemp. Math. 219, 93-110 (1998). arXiv:hep-th/9712226

10. A. Danehkar, On the cohomological derivation of Yang-Mills theory in the antifield formalism. J. High Energy Phys. Gravit. Cosmol. $\mathbf{0 3}(02), 75808$ (2017)

11. G. Barnich, N. Boulanger, A note on local BRST cohomology of Yang-Mills type theories with Abelian factor. J. Math. Phys. 59, 052302 (2018). arXiv:1802.03619 [hep-th]

12. N. Boulanger, S. Leclercq, Consistent coupling between spin- 2 and spin-3 massless fields. JHEP 11, 034 (2006). arXiv:hep-th/0609221

13. N. Boulanger, S. Leclercq, P. Sundel, On the uniqueness of minimal coupling in higher-spin gauge theory. JHEP 08, 056 (2008). arXiv:0805.2764 [hep-th]

14. M. Sakaguchi, H. Suzuki, On the interacting higher spin bosonic gauge fields in BRST-antifield formalism. Prog. Theor. Exp. Phys. (2015). arXiv:2011.02689 [hep-th]

15. S.J. Gates, M.T. Grisaru, M. Roćek, W. Siegel, Superspace (Benjamin/Cummings, Reading, 1983). arXiv:hep-th/0108200

16. I.L. Buchbinder, S.M. Kuzenko, Ideas and Methods of Supersymmetry and Supergravity (IOP Publishing, Bristol, 1998) 
17. A.S. Galperin, E.A. Ivanov, V.I. Ogievetsky, E.S. Sokatchev, Harmonic Superspace (Cambridge University Press, Cambridge, 2001)

18. C. Becchi, A. Rouet, R. Stora, The abelian Higgs Kibble Model, unitarity of the $S$-operator. Phys. Lett. B 52, 344 (1974)

19. I.V. Tyutin, Gauge invariance in field theory and statistical physics in operator formalism, Lebedev Institute preprint No. 39 (1975). arXiv:0812.0580 [hep-th]
20. L.D. Faddeev, V.N. Popov, Feynman diagrams for the Yang-Mills field. Phys. Lett. B 25, 29 (1967) 